\title{
CMOS Differential-Mode Exponential Voltage-To-Current Converter
}

\author{
WEIHSING LIU, ${ }^{1}$ SHEN-IUAN LIU ${ }^{1, *}$ AND SHUI-KEN WEI ${ }^{2}$ \\ ${ }^{1}$ Department of Electrical Engineering and Graduate Institute of Electronics Engineering, National Taiwan University, Taipei, Taiwan 10617, R.O.C. \\ ${ }^{2}$ Department of Electronic Engineering, Tung Nan Institute of Technology, Taipei, Taiwan 22202, R. O. C. \\ E-mail: lsi@cc.ee.ntu.edu.tw
}

Received May 26, 2003; Revised February 9, 2004; Accepted October 19, 2004

\begin{abstract}
A CMOS differential-mode exponential voltage-to-current converter is presented which is based on the approximated Taylor's series expansion. The proposed circuit has been fabricated in a $0.5 \mu \mathrm{m} N$-well CMOS process. Experimental results show that the output dynamic range of the proposed differential-mode exponential voltage-to-current converter can be $15 \mathrm{~dB}$ while the nonlinear error is less than $1.35 \%$. The experimental results are given to demonstrate the proposed circuits.
\end{abstract}

Key Words: CMOS, differential, exponential circuit

\section{Introduction}

The exponential function generator is one of the important building blocks in a variable gain amplifier (VGA) [1-3]. To realize the exponential function, the approximated Taylor's series expansion [4-6] and a pseudo-exponential function [7, 8] can be utilized. A differential-mode exponential voltage-to-current converter can realize the following equation,

$$
I_{\text {out }}=\exp \left(V_{1}-V_{2}\right)
$$

where $\left(V_{1}-V_{2}\right)$ is the voltage difference between two input signals and $I_{\text {out }}$ is the output current. Among [4-8], although some of them can operate with a voltage-mode input signal, however, it is limited to be a single-ended one. Since $\exp (x-$ $y) \neq \exp (x)-\exp (y)$, a differential-mode exponential voltage-to-current converter can not be realized by directly using two single-ended exponential voltageto-current converters as the input stage. For example, there are two logarithmic current-to-voltage converters with the input currents, $I_{1}$ and $I_{2}$, and the corresponding output voltages, $V_{\text {out1 }}$ and $V_{\text {out2 }}$, where $V_{\text {out1 }}=k \ln I_{1}$ and $V_{\text {out2 }}=k \ln I_{2}$, respectively, and $k$ is a constant. The ratio of the currents $I_{1}$ and $I_{2}$

*Corresponding author. can be obtained by connecting the differential-mode exponential voltage-to-current converter to the outputs of the logarithmic current-to-voltage-converters, i.e.,

$$
\begin{aligned}
I_{\text {out }} & =\exp \left(V_{\text {out } 1}-V_{\text {out } 2}\right)=\exp \left(k \ln I_{1}-k \ln I_{2}\right) \\
& =\left(\frac{I_{1}}{I_{2}}\right)^{k}
\end{aligned}
$$

Therefore, the development of the differential-mode exponential voltage-to-current converter is of certain importance. In this paper, a differential-mode exponential voltage-to-current converter is presented. It is based on the approximated Taylor's series expansion. The proposed circuit has been fabricated in a $0.5 \mu \mathrm{m}$ $\mathrm{N}$-well CMOS process and the experimental results are given to demonstrate the proposed circuit.

\section{Circuit Description}

According to the Taylor's series expansion, a general exponential function can be expressed as

$$
\exp (a x) \approx \frac{a^{2} x^{2}}{2}+a x+1 ; \quad \text { if }|a x| \ll 1
$$

The proposed differential-mode exponential voltage-to-current converter is shown in Fig. 1. Assume 


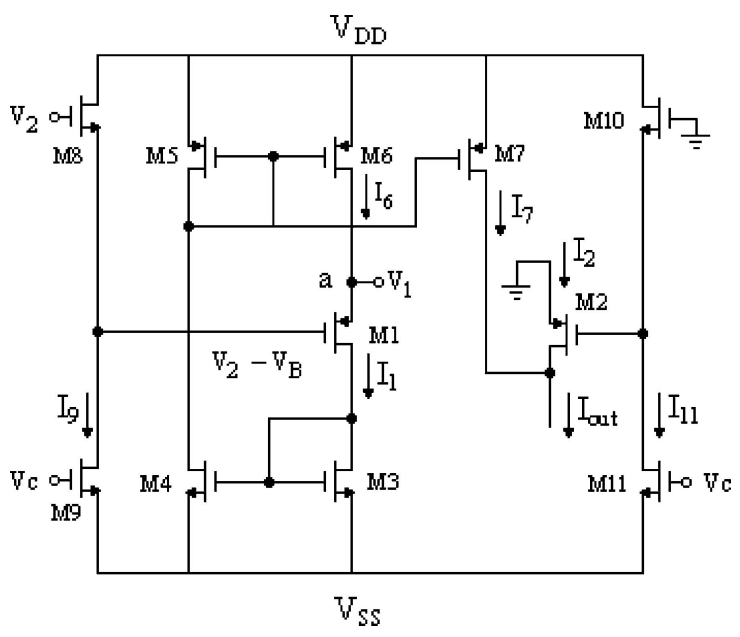

Fig. 1. Proposed differential-mode exponential voltage-to-current converter.

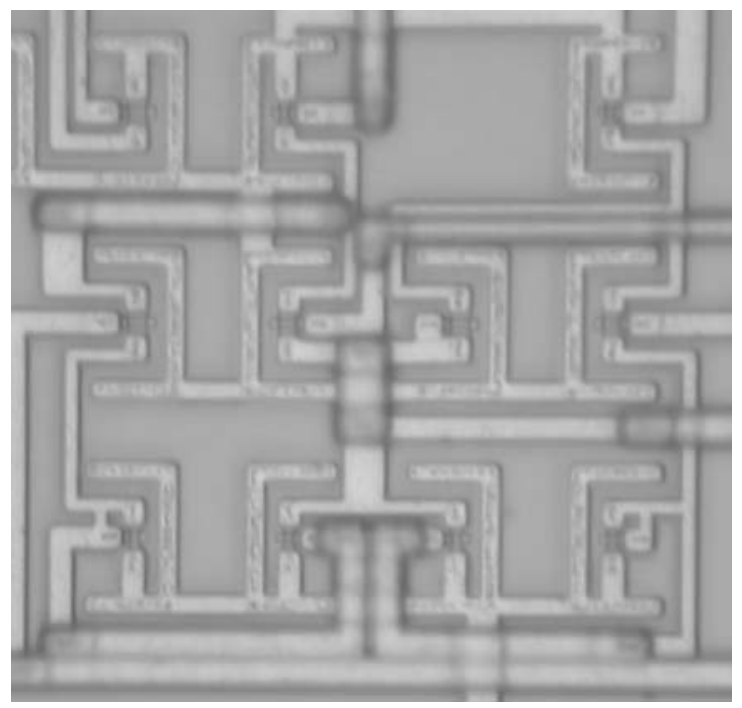

Fig. 2. The die photo of the proposed differential-mode exponential voltage-to-current converter.

that all the transistors in Fig. 1 are in saturation region without body effect. M8, M9 and M10, M11 are two level shifters. Supposed that M8-M11 are perfectly matched with a constant voltage $V_{\mathrm{c}}$, one can obtain

$$
V_{\mathrm{GS} 8}=V_{\mathrm{GS} 9}=V_{\mathrm{GS} 10}=\sqrt{\frac{2 I}{k_{n}}}+V_{T n} \triangleq V_{B}
$$

where $k_{n}=\mu_{n} C_{\mathrm{ox}} \frac{W}{L}$ is the transconductance parameter, $V_{T n}$ is the threshold voltage of M8-M11 and

$$
I_{9}=I_{11}=\frac{1}{2} k_{n}\left(V_{C}-V_{S S}-V_{T n}\right)^{2} \triangleq I,
$$

respectively. Again, assume that M1 and M2 are perfectly matched. According to the square-law characteristics of the MOSFET, one can obtain

$$
I_{1}=\frac{1}{2} k_{p}\left(V_{1}-\left(V_{2}-V_{B}\right)-\left|V_{T p}\right|\right)^{2}
$$

and

$$
I_{2}=\frac{1}{2} k_{p}\left(V_{B}-\left|V_{T p}\right|\right)^{2}
$$

where $k_{P}=\mu_{p} C_{\mathrm{ox}} \frac{W}{L}$ is the transconductance parameter and $V_{T p}$ is the threshold voltage of M1 and M2, respectively. Since M3, M4 and M5-M7 realize two current mirrors, one can obtain

$$
I_{1}=I_{6}=I_{7}
$$

Since $I_{6}=I_{1}$, there is no current to flow into the node "a". From equations (5)-(7), the output current of Fig. 1 can be given as

$$
\begin{aligned}
I_{\text {out }}= & I_{7}+I_{2}=I_{1}+I_{2}=k_{p}\left(V_{B}-\left|V_{T p}\right|\right)^{2} \\
& \times\left(\frac{\left(V_{1}-V_{2}\right)^{2}}{2\left(V_{B}-\left|V_{T p}\right|\right)^{2}}+\frac{\left(V_{1}-V_{2}\right)}{\left(V_{B}-\left|V_{T p}\right|\right)}+1\right)
\end{aligned}
$$

According to equation (2) and $V_{B}>\left|V_{T p}\right|$, equation (8) can be written as

$$
I_{\text {out }} \approx I_{b} \cdot \exp \left(a\left(V_{1}-V_{2}\right)\right)
$$

where $I_{b}=k_{p}\left(V_{B}-|V T p|\right)^{2}$ and $a=\frac{1}{V_{B}-\left|V_{T p}\right|}$. From equation (9), a differential-mode exponential voltageto-current converter can be obtained. Since M1 is assumed to be in saturation region, the input signal should be limited by

$$
V_{1}-V_{2} \geq\left|V_{T p}\right|-V_{B}
$$

and

$$
V_{2} \geq V_{B}+V_{S S}+V_{T n}-\left|V_{T p}\right|
$$

Assume that the transconductance parameters of M1 and M2 are mismatched due to the process variations, 


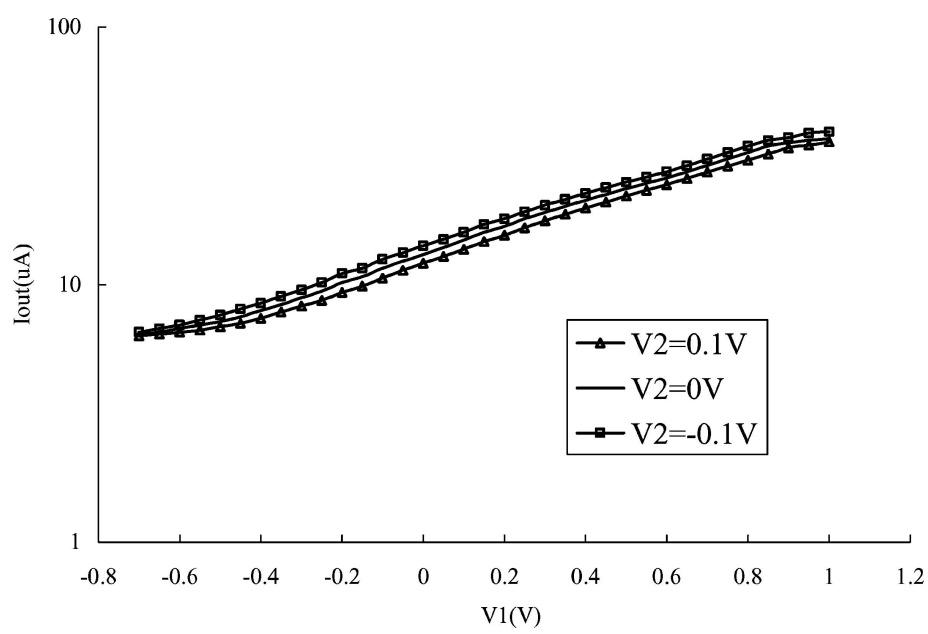

(a)

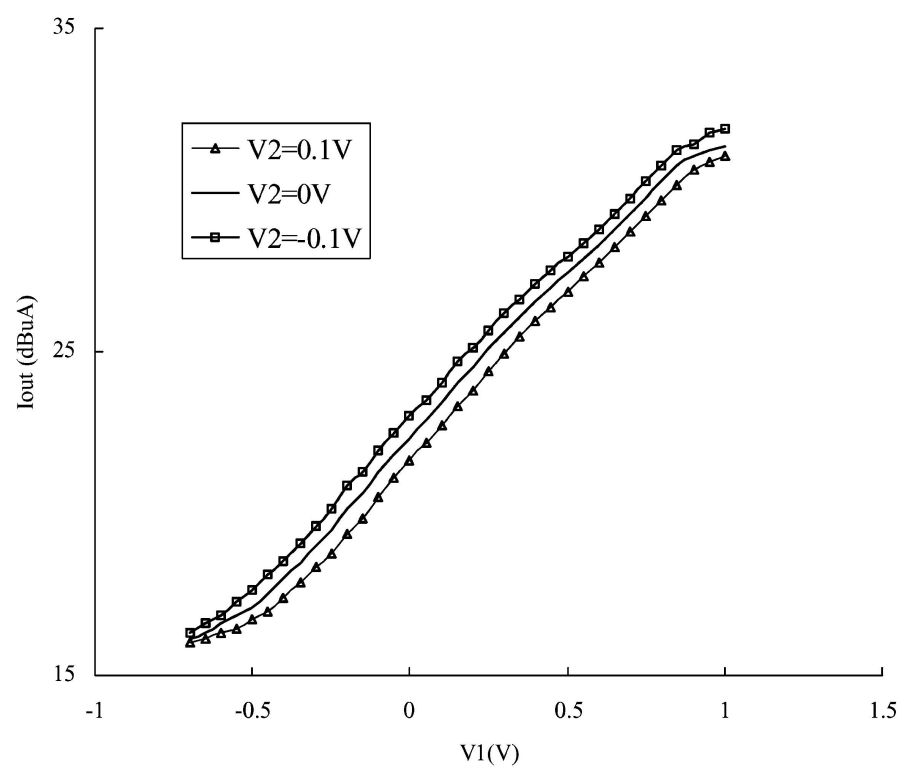

(b)

Fig. 3. Experimental results of the proposed differential-mode exponential voltage-to-current converter. (a) semi-logarithmic scale and (b) dB scale.

i.e., $k_{p 1}=k_{p}$ and $k_{p 2}=k_{p}+\Delta k_{p}$. According to equations (5) and (6), equations (8) can be rewritten as

$$
\begin{aligned}
I_{\mathrm{out}}= & k_{p}\left(V_{B}-\left|V_{T p}\right|\right)^{2}\left(\frac{\left(V_{1}-V_{2}\right)^{2}}{2\left(V_{B}-\left|V_{T p}\right|\right)^{2}}\right. \\
& \left.+\frac{\left(V_{1}-V_{2}\right)}{\left(V_{B}-\left|V_{T p}\right|\right)}+1\right)+\frac{\Delta k_{p}}{2}\left(V_{B}-\left|V_{T p}\right|\right)^{2}
\end{aligned}
$$

where an additional constant current term is generated and it will result in the offset current with re- spect to pseudo-exponential function. Suppose that the threshold voltages of M1 and M2 are not equal, i.e., $\left|V_{T p 1}\right|=\left|V_{T p}\right|$ and $\left|V_{T p 2}\right|=\left|V_{T p}\right|+\Delta V_{T p}$. From equations (5), (6)and (8) can be rewritten as

$$
\begin{aligned}
I_{\mathrm{out}}= & k_{p}\left(V_{B}-\left|V_{T p}\right|\right)^{2}\left(\frac{\left(V_{1}-V_{2}\right)^{2}}{2\left(V_{B}-\left|V_{T p}\right|\right)^{2}}\right. \\
& \left.+\frac{\left(V_{1}-V_{2}\right)}{\left(V_{B}-\left|V_{T p}\right|\right)}+1\right)+\frac{\Delta k_{p}}{2}\left(\Delta\left|V_{T p}\right|^{2}\right. \\
& \left.-2 \Delta\left|V_{T p}\right| \cdot\left(V_{B}-\left|V_{T p}\right|\right)\right)
\end{aligned}
$$


From equation (13), an additional constant current term is also generated. To avoid the body effect, all the PMOS transistors can be embodied in their individual wells and the sources of the NMOS transistors should be connected to the negative supply voltage. However, in the proposed circuit, M8 and M10 may be affected by the body effect. Assume that the threshold voltages of M8 and M10 are not equal due to the body effect, i.e., $V_{T n 8}=V_{T n}$ and $V_{T n 10}=V_{T n}+\Delta V_{T n}$. From equation (3), it will result in $V_{\mathrm{GS} 8} \neq V_{\mathrm{GS} 10}$, i.e., $V_{\mathrm{GS} 8}=V_{B}$ and $V_{\mathrm{GS} 10}=V_{B}+\Delta V_{B}$. According to equations (5), (6), and (8) can be rewritten as

$$
\begin{aligned}
I_{\text {out }}= & k_{p}\left(V_{B}-\left|V_{T p}\right|\right)^{2}\left(\frac{\left(V_{1}-V_{2}\right)^{2}}{2\left(V_{B}-\left|V_{T p}\right|\right)^{2}}\right. \\
& \left.+\frac{\left(V_{1}-V_{2}\right)}{\left(V_{B}-\left|V_{T p}\right|\right)}+1\right) \\
& +\frac{k_{p}}{2}\left(2 \Delta V_{B}\left(V_{B}-\left|V_{T p}\right|\right)+\Delta V_{B}^{2}\right)
\end{aligned}
$$

From equation (14), an unexpected offset current is generated. In order to reduce the offset currents in equations (12)-(14), careful layout is necessary. If the offset currents are significant, long channel devices for M1 and M2 can be used to reduce the currents.

Finally, the Monte Carlo analysis shows that, as a $10 \%$ variation of the transconductance parameter and the threshold voltage of M1 and M2, after 30 times iterations, the variations of the output voltage are $0.207 \%$ and $-0.243 \%$, respectively. Also, for simulating the body effect, assume that there is $10 \%$ variation of the threshold voltage of M8 and M10, the Monte Carlo analysis shows that, the corresponding variations of the output voltage is $0.246 \%$.

\section{Experimental Results}

The proposed differential-mode exponential voltageto-current converter has been fabricated in a $0.5 \mu \mathrm{m}$ $\mathrm{N}$-well CMOS process. The die photograph is shown in Fig. 2. The aspect ratios of the transistors of the proposed circuit in Fig. 1 are all $\left(\frac{W}{L}\right)=\left(\frac{1 \mu \mathrm{m}}{1 \mu \mathrm{m}}\right)$. To test the proposed differential-mode exponential voltage-tocurrent converter, the experiment were performed with the supply voltages $V_{D D}=\left|V_{S S}\right|=2.5 \mathrm{~V}$ and the constant voltage $V_{c}=-0.75 \mathrm{~V}$. As the input voltage $V_{1}$ varies from $-0.5 \mathrm{~V}$ to $0.85 \mathrm{~V}$, the input voltage $V_{2}$ was set to $-0.1 \mathrm{~V}, 0 \mathrm{~V}$ and $0.1 \mathrm{~V}$, respectively. The measured results are shown in Fig. 3(a) and (b). According to Fig.

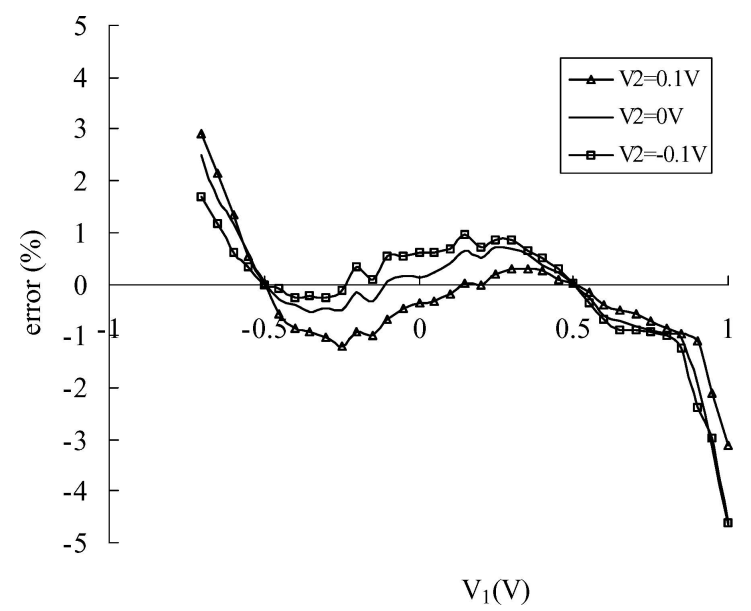

Fig. 4. Measured errors between the measured results and the theoretical values of the proposed differential-mode exponential voltageto-current converter.

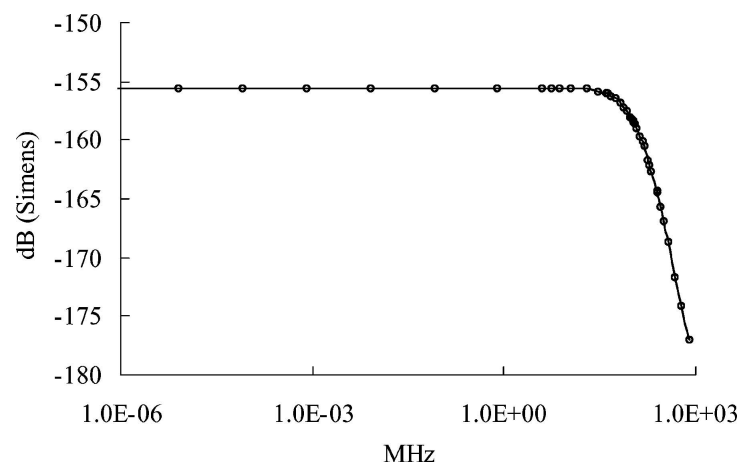

Fig. 5. Measured frequency response of the proposed differentialmode exponential voltage-to-current converter.

3(b), all the measured output currents exhibit a linear range of about $15 \mathrm{~dB}$. Figure 4 shows the errors between the measured results and the theoretical values calculated by equation (9). For a $15 \mathrm{~dB}$ linear output range, the maximum measured errors for the different input considerations were $1.35 \%$ (for $V_{2}=0.1 \mathrm{~V}$ ), $1.15 \%$ (for $V_{2}=0 \mathrm{~V}$ ) and $1.16 \%$ (for $V_{2}=-0.1 \mathrm{~V}$ ), respectively. However, most the measured errors are less than $1 \%$. Also the measured maximum power consumption is less than $0.96 \mathrm{~mW}$ (at $V_{1}=0.25 \mathrm{~V}, V_{2}=-0.1 \mathrm{~V}$ ). The frequency response is shown in Fig. 5 which was measured under the conditions of the dc input voltage $V_{1}=0.3 \mathrm{~V}$, ac input voltage $V_{1}=0.03 \mathrm{~V}$ and the dc input voltage $V_{2}=0 \mathrm{~V}$, respectively. For a $10 \mathrm{~K} \Omega$ resistor load, the measured result shows that the corresponding $-3 \mathrm{~dB}$ bandwidth can be $111.2 \mathrm{MHz}$. 


\section{Conclusion}

In this paper, a CMOS differential-mode exponential voltage-to-current converter has been developed. Experimental results have been given to confirm the validity of the theoretical analysis. The proposed circuit is expected to be useful in the design of VGA and other analog signal processing applications.

\section{Acknowledgment}

This work is partially supported by the National Science Council, Taiwan, Republic of China under Grant NSC 91-2626-E-236-002. The author Weihsing Liu is also with the faculty of the Department of Electronic Engineering, Tung Nan Institute of Technology.

\section{References}

1. A. Motamed, C. Hwang, and M. Ismail, "A low-voltage lowpower wide range CMOS variable gain amplifier.' IEEE Trans. on Circuits Systems-II: Analog and Digital Signal Processing, vol. 45 , no.7, pp. 800-810, 1998 .

2. C. Lin, T. Pimenta, and M. Ismail, "Universal exponential function implementation using highly-linear CMOS V-I converters for dB-linear (AGC) applications," in Proc. 1998 Midwest Symposium on Circuits and Systems, 1999, pp. 360363.

3. C.C. Chang, M. Lin, and S.I. Liu, "CMOS current-mode exponential-control variable-gain amplifier." Electronics Letters, vol. 37, pp. 868-869, 2001.

4. C.C. Chang and S.I. Liu, "Pseudo-exponential function using MOSFETs in saturation." IEEE Trans. Circuits and Systems-II: Analog and Digital Signal Processing, vol. 47, pp. 1318-1321, 2000.

5. W. Liu, C.C. Chang, and S.I. Liu, "Realization of exponential V-I converter using composite NMOS transistors." Electronics. Letters, vol. 36, no. 1, pp. 8-10, 2000.

6. S. Vlassis, "CMOS current-mode pseudo-exponential function circuit." Electronics Letters, vol. 37, no. 8, pp. 471-472, 2001.

7. A. Motamed and M. Ismail, "CMOS exponential current-tovoltage converter.” Electronics Letters, vol. 33, no. 12, pp. 9981000, 1997.

8. W. Liu and S.I. Liu, "CMOS exponential function generator." Electronics Letters, vol. 39, no.1, pp. 1-2, 2003.

9. E.A.Vittoz, "Micropower techniques," in Design of MOS VLSI Circuits for Telecommunications, edited by J. France and Y. Tsivids, Printice Hall, 1994.

10. E. Vittoz and J. Fellrath, "CMOS analog integrated circuits based on weak inversion operations." IEEE Journal of Solid-State Circuits, vol. 12, no. 3, pp. 224-231, 1977.

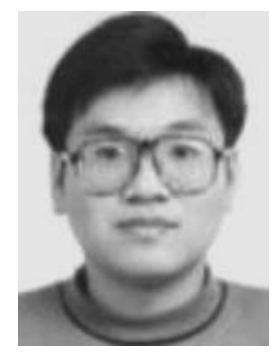

Shen-Iuan Liu was born in Keelung, Taiwan, Republic of China, 1965. He received both the B.S. and $\mathrm{Ph} . \mathrm{D}$. degree in electrical engineering from National Taiwan University (NTU), Taipei, in 1987 and 1991, respectively.

During 1991-1993 he served as a second lieutenant in Chinese Air Force. During 1991-1994, he was an Associate Professor in the Department of Electronic Engineering of National Taiwan Institute of Technology. He joined in the Department of Electrical Engineering, NTU, Taipei, Taiwan in 1994 and he has been the Professor since 1998. He obtained the Engineering Paper Award from the Chinese Institute of Engineers, 2003. He obtained the Young Professor Teaching Award from MXIC Inc. and the Research Achievement Award from NTU, 2004. His research interests are in analog and digital integrated circuits and systems.

Dr. Liu has served a chair on IEEE SSCS Taipei Chapter from 2004. Dr. Liu has served a general chair on the 15th VLSI Design/CAD symposium, Taiwan, 2004 and a Program Co-chair on the Fourth IEEE AsiaPacific Conference on Advanced System Integrated Circuits, Japan, 2004. He is a senior member of IEEE.

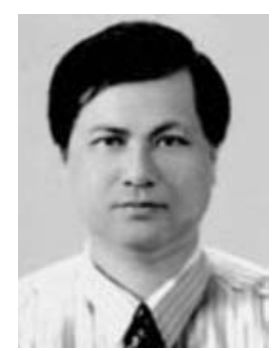

Shui-Ken Wei was born in Yun-Lin, Taiwan, Republic of China, 1955. He received the M.S. degree in Northrop University, California, U.S.A. in 1986. He joined in the faculty of the Department of Electronic Engineering, Tung Nan Institute of Technology, Taipei, Taiwan in 1979 and he has been an associate professor since 1998. From 1999 on, he has also been the head of 
the Department of Electronic Engineering, Tung Nan Institute of Technology. His research interests are in computer-aided circuits design and the design and applications of multimedia and microcomputer systems.

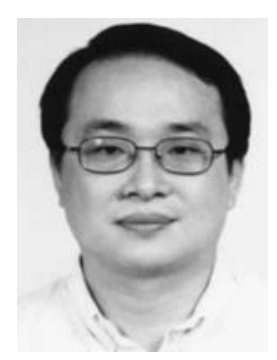

Weihsing Liu was born in Taipei, Taiwan, Republic of China, 1963. He received the B.S. degree in electronic engineering from Chung-Yuan Christian University, Chung-Li, Taiwan in 1986 and the M.S. and $\mathrm{Ph} . \mathrm{D}$. degrees in electrical engineering from Pennsylvania state university, Pennsylvania, U.S.A. and National Taiwan University, Taipei, Taiwan in 1991 and 2004, respectively. After he received the M.S. degree, he jointed in the faculty of the Department of Electronic Engineering, Tung Nan Institute of Technology, Taipei, Taiwan. His research interests focuses on analog and digital integrated circuits design. 